$$
\text { CONF- } 970324--6
$$

\title{
Active chatter control in a milling machine
}

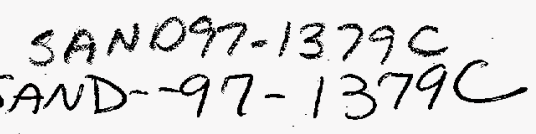

J. L. Dohner ${ }^{a}$, T.D. Hinnerichs ${ }^{a}$, J. P. Lauffer ${ }^{\mathrm{a}}$, C.M. Kwan ${ }^{\mathrm{b}}$, M.E. Regelbrugge ${ }^{\mathrm{c}}$, N. Shankar ${ }^{\mathrm{c}}$

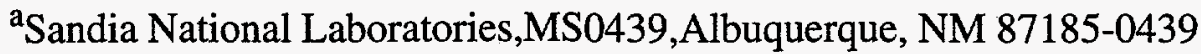

bIntelligent Automation Inc., 2 Research Place, Rockville, MD 20850

${ }^{\mathrm{c}}$ Lockheed Martin ATC, 3251 Hanover St., Palo Alto, CA 94304-1191

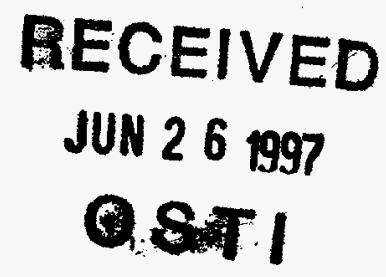

\begin{abstract}
The use of active feedback compensation to mitigate cutting instabilities in an advanced milling machine is discussed in this paper. A linear structural model delineating dynamics significant to the onset of cutting instabilities was combined with a nonlinear cutting model to form a dynamic depiction of an existing milling machine. The model was validated with experimental data. Modifications made to an existing machine model were used to predict alterations in dynamics due to the integration of active feedback compensation. From simulations, subcomponent requirements were evaluated and cutting enhancements were predicted. Active compensation was shown to enable more than double the metal removal rate over conventional milling machines.
\end{abstract}

Keywords: milling, controls, chatter

\subsection{INTRODUCTION}

Maximum metal removal rate is a quantitative measure of the productive capacity of a machine tool. This measure, which is machine as well as tool dependent, is limited by the onset of machining instabilities ${ }^{1,2,3,4}$. Machining instabilities are minimized by enhancing the stability of structural vibratory modes significant to cutting dynamics.

Active methods can be used to enhance the stability of pertinent structural vibratory modes for a variety of machining configurations. Although active methods are relatively immature ${ }^{5}$, potential performance enhancements resulting from active implementation overshadow performance enhancements resulting from current passive innovations.

Active methods can be categorized into active/passive, process control, and feedback compensation methods. Active/passive methods actively tune or mimic stabilizing passive absorbers ${ }^{6,7,8}$. Process control methods alter or modulate operating parameters (spindle speed, feed rate, etc.) to locate, or oscillate between, predetermined stability domains $\mathrm{s}^{9,10}$, and feedback compensation methods actively modify machine dynamics such that domains of stability are enlarged ${ }^{11,12,13}$.

Presented within this paper is the design of an active feedback compensator which will actively modify machine dynamics such that domains of stability are enlarged. This design process entails;

1) development and validation of a model,

2) modification of the model for active compensation, and

3) performance evaluation.

In section 2, modeling and validation is discussed. In section 3, the modeling of dynamics essential for active compensation is discussed. And in section 4, performance earnings are presented.

\subsection{MODEL DEVELOPMENT}

A machine model is a set of mathematical relationships relating dynamic response parameters (displacement of tool tip center, force on tool, etc...) to a set of process parameters (spindle speed, number of teeth on tool, etc...). The goal of modeling is to create mathematical relationships which are sufficiently accurate for the purpose of prediction. Model accuracy is quantified by comparison with experimental data. The model discussed in this section is shown to compare well with experimental data.

The machine model is the unification of a cutting model and a structural model. The cutting model describes the interaction of the tool and the workpiece, whereas, the structural model describes the response of the tool due to tool/workpiece interaction. 


\section{DISCLAMIER}

Portions of this doenment may be illegible in electronic image products. Imsges are produced from the best available original documert. 
The cutting model consists of a set of mathematical relationships which describe tool tip force $\vec{f}_{t}$, as a function of tool tip displacement $\vec{d}_{t}$, whereas the structural model consists of a set of mathematical relationships which describe $\vec{d}_{t}$ and its time derivatives as a function of $\vec{f}_{t}$. As shown in Figure 1, the machine model is the structural dynamics model in feedback with the cutting model.

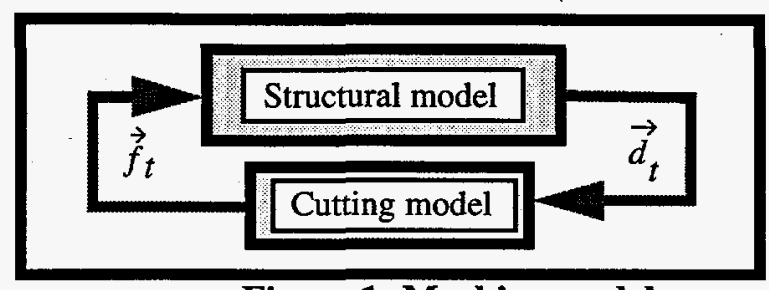

Figure 1: Machine model

\subsection{A cutting model}

In this section, a simple, but adequate, model of cutting in milling is detailed. The work presented in this section is similar to that presented by Smith and Tlusty ${ }^{14,15}$.

Figure 2a contains an illustration of a helical tool translating through a workpiece, and Figure $2 b$ contains an illustration of the Figure 2a plane in which this translation occurs. If the tool and the structure were perfectly rigid, the cutting edges of the tool

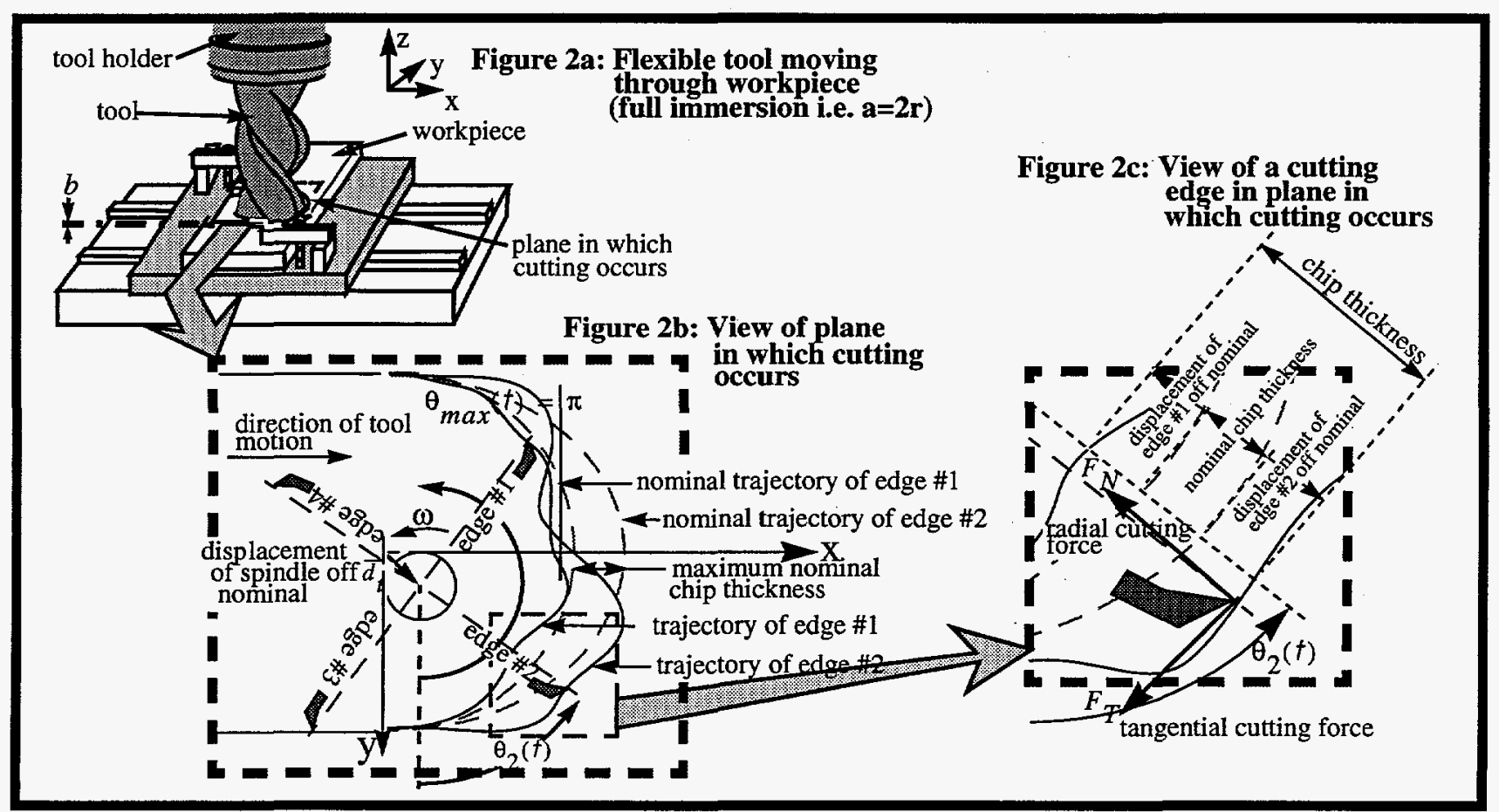

Figure 2: Cutting model detail

would move along the illustrated dashed lines. These dashed lines, called nominal trajectories, define nominal chip thicknesses, $t_{\text {nom }_{i}}$. Nominal chip thicknesses are the thicknesses of chips removed due to cutting when the tool and structure are perfectly rigid. Nominal chip thickness at the $i^{\text {th }}$ edge can be approximated by

$$
t_{n o m_{i}}=t_{\max } \sin \left(\theta_{i}(t)\right) \text { for }(n-1) \pi \leq \theta_{i}(t) \leq(n-1) \pi+\theta_{\max }
$$


where $n=1,3,5 \ldots ; t_{\max }=\left(\frac{2 \pi}{N_{t} \omega}\right) f$ is called the chip loading; $i=1,2,3 \ldots N_{t} ; N_{t}$ is the number of teeth per tool; $\omega$ is the rotational speed of the tool (in rads/sec); $\theta_{i}(t)$ is the rotational location of the $i^{\text {th }}$ cutting edge at time $t ; f$ is the feed rate of the tool through the workpiece (in length/sec) and $\theta_{\max }$ is the maximum relative rotational angle for which a cutting edge contacts the workpiece. $\theta_{\max }=\operatorname{acos}(1-a / r)$ where $a$ is the immersion depth of the tool into the workpiece and where $r$ is the radius of the tool.

Since the tool and the structure are never perfectly rigid, tool edges never follow nominal trajectories. If $\hat{i}$ and $\hat{j}$ are unit vectors in the $\mathrm{x}$ and $\mathrm{y}$ directions, and if $\vec{d}_{t}=d_{x} \hat{i}+d_{y} \hat{j}$ is the vibratory displacement at tool tip center, then radial variations of displacement off nominal at the $i^{\text {th }}$ cutting edge are given by

$$
z_{i}\left(\theta_{i}(t)\right)=\vec{d}_{t} \cdot\left[\sin \left(\theta_{i}(t)\right) \hat{i}+\cos \left(\theta_{i}(t)\right) \hat{j}\right]
$$

If the $i-1^{\text {th }}$ cutting edge is sequential and leading in rotation to the $i^{\text {th }}$ cutting edge, chip thickness, $t_{i}$ at the $i^{\text {th }}$ edge is given by

$$
t_{i}=t_{n o m_{i}}+z_{i}\left(\theta_{i}(t)\right)-z_{i-1}\left(\theta_{i}(t)\right)
$$

where $\theta_{i}(t)=\theta_{i-1}\left(t-\frac{2 \pi}{N_{t} \omega}\right)$

Force on the tool tip, $\vec{f}_{t}$, is given by

$$
\begin{gathered}
\vec{f}_{t}=\sum_{i=1}^{N_{t}} \vec{f}_{t_{i}} \\
\vec{f}_{t_{i}}=f_{x_{i} \hat{i}+f_{y_{i}} \hat{j},} \\
f_{x_{i}}=-F_{N_{i}} \sin \left(\theta_{i}(t)\right)-F_{T_{i}} \cos \left(\theta_{i}(t)\right), \\
f_{y_{i}}=-F_{N_{i}} \cos \left(\theta_{i}(t)\right)+F_{T_{i}} \sin \left(\theta_{i}(t)\right), \\
F_{N_{i}}=K_{a} F_{T_{i}}, \text { and }, F_{T_{i}}=K_{s} t_{i} b+\frac{\alpha}{\omega \partial t} t_{i}
\end{gathered}
$$

where $K_{s}$ is called the specific cutting stiffness (a material property), $K_{a} \approx 0.3 K_{s}$ for most applications, $b$ is depth of cut (shown in Figure 1a) and $\alpha$ is a process damping coefficient which approximates low- $\omega$ frictional contributions related to tool flank rubbing on the workpiece.

Equations 2.1 determine chip thickness in terms of tool tip displacement, and equations 2.2 determine tool tip force in terms of chip thickness. Together, equations 2.1 and 2.2 determine tool tip force, $\vec{f}_{t}$, in terms of tool tip displacement, $\vec{d}_{t}$. Therefore, equations 2.1 and 2.2 are a cutting model. 


\subsection{A structural model}

The structural model is a set of mathematical relationships representing $\vec{d}_{t}$ and its time derivatives in terms of $\vec{f}_{t}$. This model is always machine dependent. In this paper, the Octahedral Hexapod Milling (OHM) machine shown in Figure 3 will be examined. This machine consists of a stiff outer octahedral space frame supporting six hexapod struts connected to an inner milling platform. The stiff outer frame pins one end of each hexapod strut. Hexapod struts consist of two telescoping tubes connected by a ball screw mechanism. As the screw rotates, the telescoping tubes undergo relative translations and strut length is altered. Screws are driven by motors at the pinned end of each strut. By controlling screw rotation, the length of each strut is altered and platform motion is controlled.

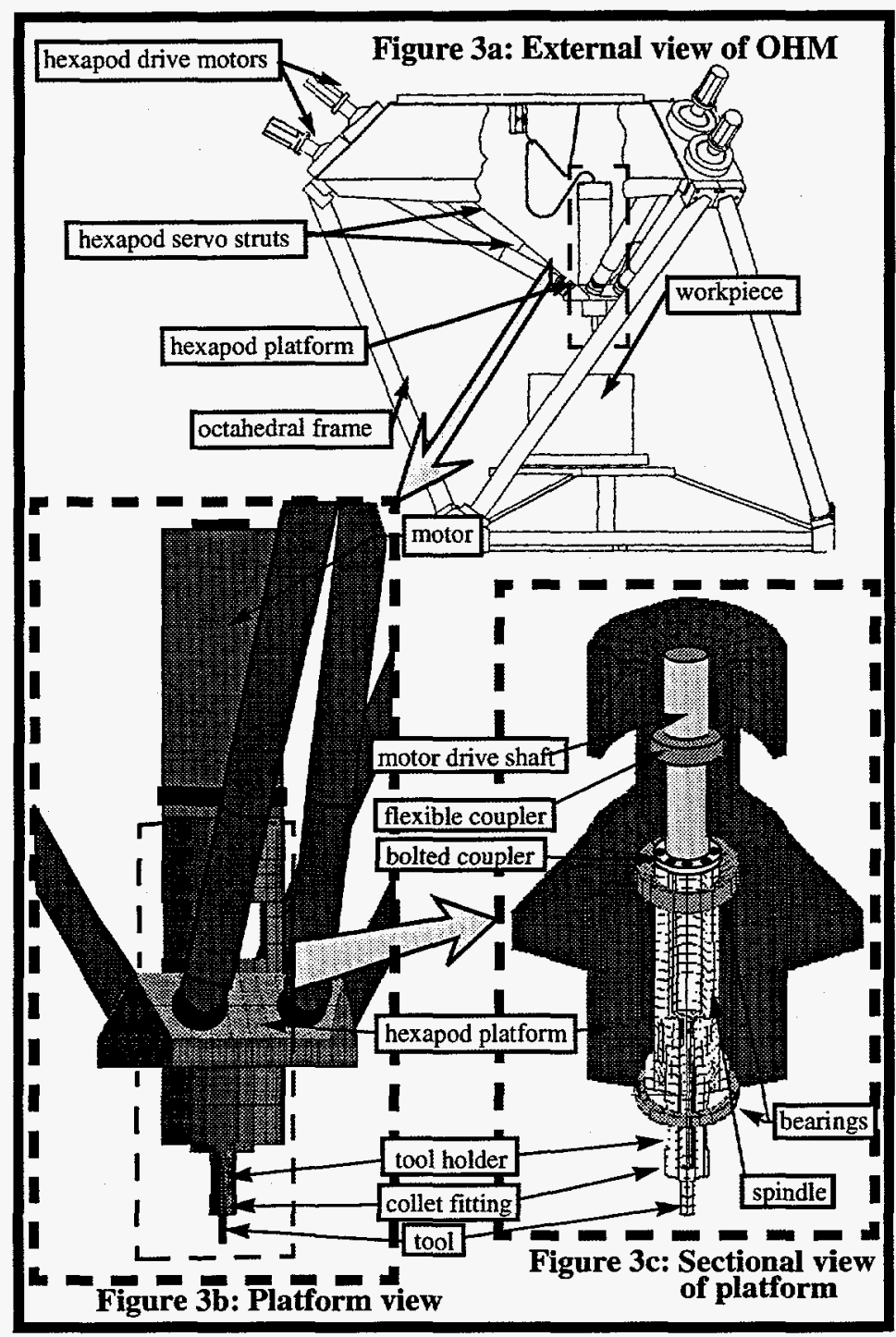

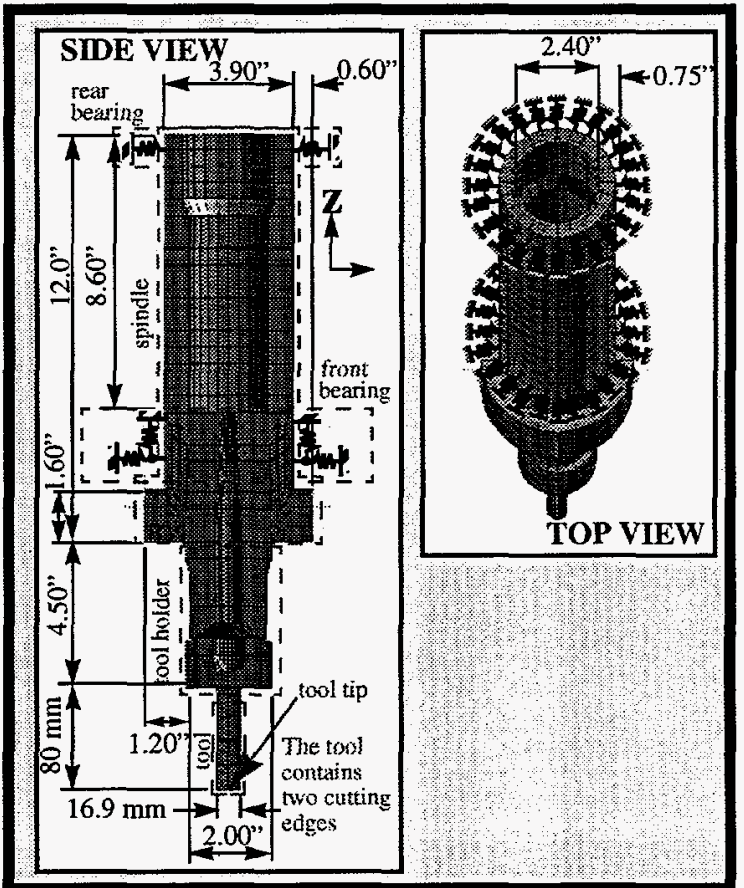

Figure 4: Finite element model of OHM machine

Figure 3: Octahedral Hexapod Milling (OHM) machine

OHM machine structural dynamics are non-linear and hysteretic due to the existence of bolted connections, compression fittings, socketed joints, and flexible couplers ${ }^{16}$. Moreover, since frequencies of interest are relatively high, linear modal dynamics are dense. Therefore, obtaining an encompassing model of machine dynamics is impossible.

Fortunately, structural modeling complexity can be reduced by making two simplifying, yet accurate, assumptions.

Assumption 1: The most flexible modes contributing to tool tip drive point compliance are those modes most likely to produce cutting instabilities. 
Assumption 2: The most flexible modes contributing to tool tip drive point compliance are those consisting of local vibrations in the tool, the tool holder, the spindle, and the bearings.

From assumptions 1) and 2), a structural model of the OHM machine sufficient for integration with the section 2.1 cutting model, need only contain the dynamics of the tool, the tool holder, the spindle, and the bearings.

A Finite Element (FE) mesh of the tool, the tool holder, the spindle, and the bearings is shown in Figure 4. This mesh was constructed in a rectangular Cartesian coordinate system with the axis of the tool, the tool holder, the spindle, and the bearings alined with the $\mathrm{z}$ axis.

The second order matrix equation resulting from the Figure $4 \mathrm{FE}$ mesh contained 8661 physical Degrees Of Freedom (DOF). These DOF were reduced to 3 physical DOF and 13 generalized DOF using component mode synthesis ${ }^{17}$. The reduced second order model was then shifted to first order block diagonal form and reduced further via modal cost analysis ${ }^{18}$.The final, reduced order model contained 16 states and took the form

$$
\begin{gathered}
\frac{d \mathbf{X}_{u}}{d t}=\mathbf{A}_{u} \mathbf{X}_{u}+\mathbf{B}_{u_{t}} \mathbf{f}_{t} \\
\text { and } \mathbf{d}_{t}=\mathbf{C} \mathbf{X}_{u} \\
\text { where } \mathbf{f}_{t}=\left[f_{x} f_{y}\right]^{T}, \mathbf{d}_{t}=\left[d_{x} d_{y}\right]^{T}
\end{gathered}
$$

and $\mathbf{X}_{u}$ is a state vector.

Notice that equations 2.3 are a set of mathematical relationships relating displacement at the tool tip, $\mathbf{d}_{t}$, to force at the tool tip,

$\mathbf{f}_{t}$. Therefore, they are a structural model.

\subsection{Model validation}

In section 2.1 a cutting model was presented, and in section 2.2 a structural model was presented. The union of these two models in feedback is the machine model. The quality of modeling was determined by comparing modeled dynamics to experimental data.

Modeled and measured tool tip, drive point compliance is shown in Figure 5. Agreement between modeled data and reality is acceptable. The model predicted both natural frequencies and mode shapes to respectable levels. Modal damping, a parameter which cannot be derived theoretically, was determined experimentally and was incorporated into the model. Process damping was also determined experimentally to match cutting stability test data as shown in Figure 6 for rpm less than 4000 . The machine model is the structural model in feedback with the cutting model. The machine model can be used to predict maximum stable depths of cut for a distribution of spindle speeds. A comparison between modeled and measured maximum stable depths of cut is shown in Figure 6. These curves also agree quite well.

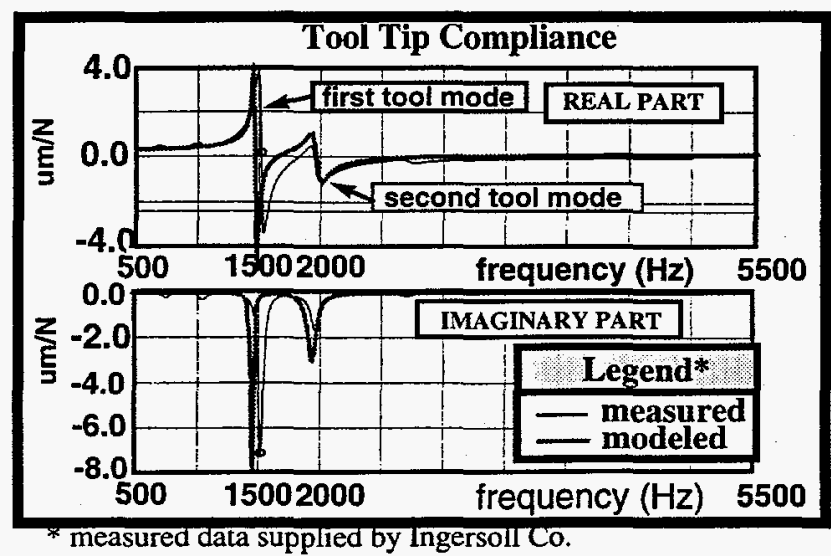

Figure 5: Modeled and measured tool tip compliance

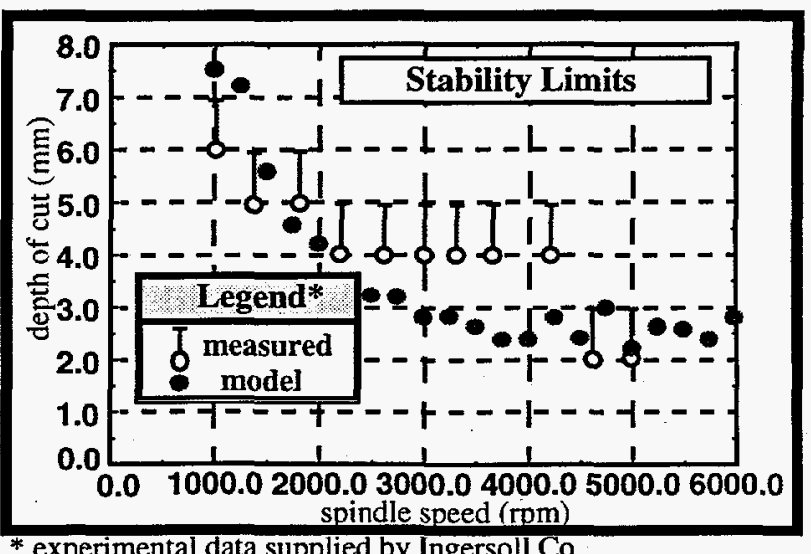

Figure 6: Modeled and measured cutting stability limits 
In the next section, the machine model will be modified to account for alterations in dynamics due to the integration of active compensation. This model will then be used to assess control feasibility as well as to estimate subcomponent requirements.

\subsection{MODEL MODIFICATION}

In Figure 7, hardware and software modifications to the Figure $3 \mathrm{FE}$ model are shown.The Figure 3, unmodified FE mesh consisted of a tool, a tool holder, a spindle and bearings. The Figure 7, modified FE mesh contains all of this, in addition to a cartridge, actuators, a pressurized oil bearing, and the platform which mounts to the hexapod struts. The cartridge is an aluminum cylinder used to minimize misalignment between mechanical bearings. It is connected at mid-section to the platform by a pressurized oil bearing. This bearing produces high stiffness along the axis of symmetry and low stiffnesses in directions perpendicular to the axis of symmetry. Actuators drive the cartridge in these low stiffness directions to cancel vibrations.

Feedback compensation is used to alter machine dynamics such that tool stiffness, and therefore metal removal rate, is enhanced. Compensation is implemented via strain sensors, a telemetry sensor package, filters, analog to digital (A/D) converters, an encoder, a processor, digital to analog (D/A) converters, power amplifiers, and actuators. Tool tip vibration is sensed by strain gages located at the root of the tool. These measurements are transmitted off the rotating spindle by frequency modulated radio transmissions which are captured by a demodulating receiver. Demodulated strain signals are then low-pass filtered, and fed into A/D converters and a processor. In the processor, strain signals are transformed from the rotating coordinate system of the tool, the tool holder and the spindle, to the non-rotating coordinate system of the platform, the cartridge, and the actuators. Relative rotation between coordinate systems is sensed by an encoder. Filtered, digitized, and translated strain signals are numerical inputs into a discrete mathematical relationship called the control law. Control law output

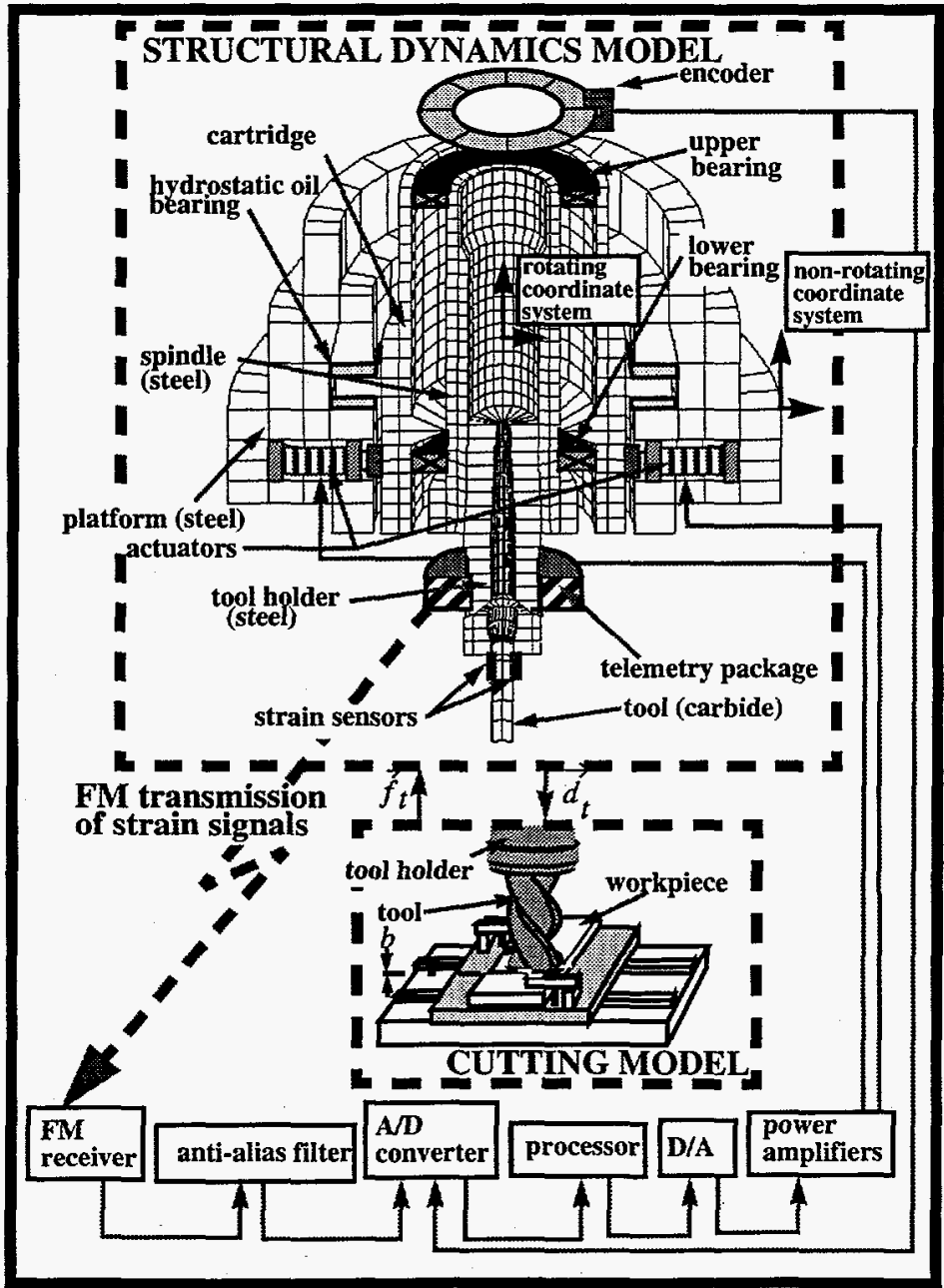

Figure 7: Modified structural dynamics model with active compensation 
is a numerical data stream which is converted into voltage signals by D/A converters. These voltages are then filtered, and fed into power amplifiers. Voltage and current from power amplifiers drive actuators which dynamically stiffen tool tip motion.

In the following subsections, subcomponent dynamics are described.

\subsection{Actuators}

An illustration of actuator architecture is shown in Figure 8a. Each actuator consists of a set of active layers, a set of end caps, a set of insulators, a single load cell, and an end alignment bearing. The set of active layers is called the stack. Two types of stacks will be discussed. The first type of stack, called a PZT stack, is constructed from the piezo-electric material, PZT $4^{19}$, and the second type of stack, called a PMN stack, is constructed from the electrostrictive material, $\mathrm{PMN}^{20}$.

Between each active layer is a conducting film which is attached to either an electrical ground, a DC bias voltage, $V_{\text {bias }}$, or a drive voltage, $V$. In Figure 8, electrical connections and layering in PZT and PMN stacks are shown.The piezo-electric, PZT4, is layered with alternating polarity and is wired such that every other conducting film is grounded or driven by driver voltage, $V$. PMN layers are wired in a similar fashion, however since PMN has no polarity, a bias voltage, $V_{\text {bias }}$, must be added to the drive voltage, $V$, to produce symmetric actuation. Both stacks are mechanically and/or electrically prestressed such that no

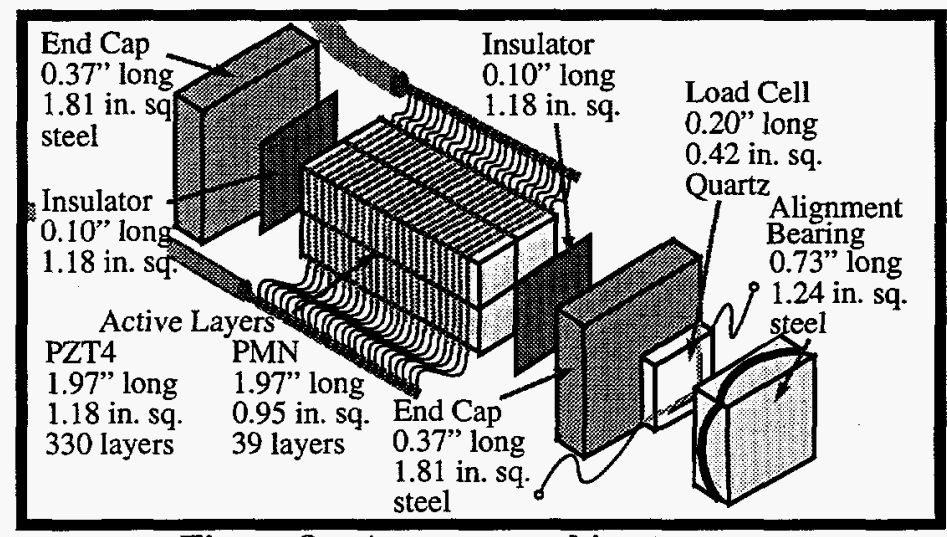

Figure 8a: Actuator architecture

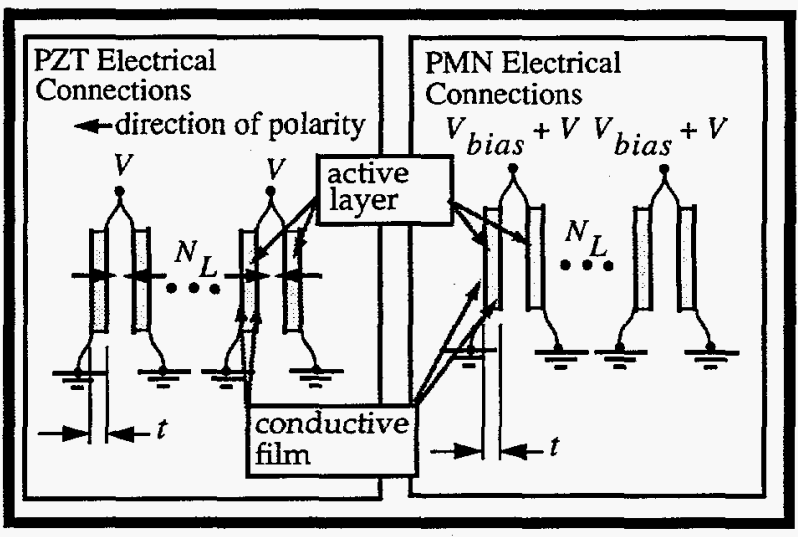

Figure 8b: Wiring for PZT and PMN layers

layer is in tension during operation.

If there are $N_{L}$ layers in the stack and the stack is $L$ long, then the thickness of each layer is $t=L / N_{L}$. If each layer is square with area $A$, and $t$ is much less than $\sqrt{A}$, then the electric field $E$, through a layer can be approximated as $E=V / t$.

Moreover, if $t$ is small compared to the dilatational wavelength $\lambda$, of the stack, $\Delta d$ is the incremental change in $t$ across a layer, and $F$ is the force through a layer, then the mechanical strain $S$, through a layer can be approximated by $S=\Delta d / t$, and the mechanical stress $T$, across a layer can be approximated by $T=F / A$.

The form of the models which delineate stress $T$, strain $S$, electric field $E$, charge displacement $D$, and current $i$, in a PZT and a PMN layer are identical when linearizing about a well chosen operating voltage. Therefore, a separate analysis for each type of stack is not needed.Only the parameters in the model need change to delineate between materials. A "three parameter" model describing layer dynamics is given by ${ }^{19,20}$

$$
\begin{gathered}
T=C^{D} S-h D, \\
E=-h S+\beta^{S} D, \\
i=A \frac{d D}{d t}
\end{gathered}
$$

where $C^{D}, h$, and $\beta^{s}$ are material parameters. Material parameters are given in Table 3.1 for both PZT4 and PMN.

Solving equation $3.1 \mathrm{~b}$ for $D$ and substituting the result into equation $3.1 \mathrm{a}$ and $3.1 \mathrm{c}$ gives 
Table 3.1 Material Parameters for PZT and PMN

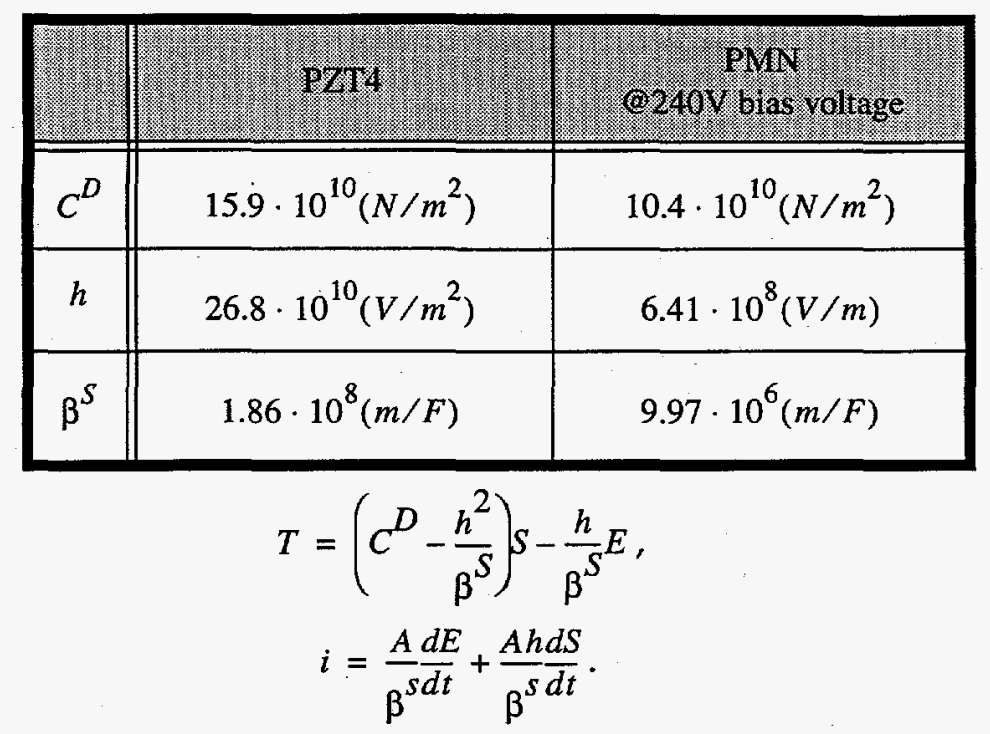

Equation 3.2 is a relationship between axial stress and strain where $Y=C^{D}-\frac{h^{2}}{\beta^{S}}$ is an effective elastic modulus and

$\frac{h}{\beta^{S}} E=\frac{h}{\beta^{S} t} V$ is a voltage induced stress. Therefore, the axial dynamics of a layer are equivalent to those of a bar of length $t$, cross sectional area $A$, and elastic modulus $Y$, with voltage dependent forces $F_{a}=\frac{A h}{\beta^{S}} V$ acting at each end. The density of this bar $\rho_{a}$, would be the same as the density of the active material.

If the dilational wavelength $\lambda$, of the stack is much greater than $L$, stress and strain through each layer can be equilibrated.

From Figure 3, the highest frequency of interest is about $3 \mathrm{kHz}$. Since $\frac{\lambda}{L}=27.1$ at $3 \mathrm{kHz}$ for PZT4 and $\frac{\lambda}{L}=18.6$ at $3 \mathrm{kHz}$ for PMN, the dilational wavelength of the stack is much greater than the length of the stack, and stress and strain through each layer are similar. Thus, the stack can be modeled as a bar of modulus $Y$, and of density $\rho_{a}$, with voltage dependent forces

$F_{a}=\frac{A}{\beta^{S}} V$, acting at each end. The current $I$, required to produce the forces $F_{a}$, is

$$
I=N_{L} i=\frac{A}{\beta^{S} t}\left(N_{L} \frac{d V}{d t}+h \frac{d}{d t} \Delta d a\right)
$$

where $\Delta d_{a}=N_{L} \Delta d$.

\subsection{Structure}

The Figure 7 structure was modeled using Finite Elements (FE). Actuators were modeled as a series of one dimensional bar elements and the hydrostatic oil bearing was modeled by using nodal constraints.

The resulting second order matrix FE model contained over 16,000 DOF. This model was reduced down to 69 DOF via component mode synthesis. Of these $69 \mathrm{DOF}, 39$ were physical and 30 were generalized. The model was then transformed into first order form and further reduced via modal cost analysis. The final first order state space solution contained 28 states and took the form 


$$
\begin{aligned}
\frac{d \mathbf{X}}{d t}=\mathbf{A}_{s} \mathbf{X}_{s}+\mathbf{B}_{t} \mathbf{f}_{t}+\mathbf{B}_{a} \mathbf{f}_{a} \\
\mathbf{d}_{a}=\mathbf{C}_{a} \mathbf{X}_{s} \\
\dot{\mathbf{d}}_{a}=\mathbf{C}_{a d} \mathbf{X}_{s} \\
\varepsilon=\mathbf{C}_{s} \mathbf{X}_{s} \\
\mathbf{d}_{t}=\mathbf{C}_{t} \mathbf{X}_{s} \\
\mathbf{f}_{m}=\mathbf{C}_{m} \mathbf{X}_{s}
\end{aligned}
$$

where $\mathbf{f}_{t}$ is a vector of tool tip forces, $\mathbf{f}_{a}$ is a vector of actuator forces, $\mathbf{d}_{t}$ is a vector of tool tip displacements, $\mathbf{d}_{a}$ is a vector of displacements across the stack, $\dot{\mathbf{d}}_{a}$ a vector of the time derivative of displacements across the stack, $\varepsilon$ is a vector of strains at the root of the tool, and $\mathbf{f}_{m}$ is a vector of load cell force measurements. All forces, displacements and strains were relative to the non-rotating reference frame of the cartridge and platform.

\subsection{Prefilters and power amplifiers}

Power amplifiers supply the voltages $V$, and the currents $I$, required to drive actuator stacks. A power amplifier attempts to produce an output signal $V$, that follows an input signal $v_{p}$, such that $V=S_{P} v_{p}$ where $S_{P}$ is the amplifier gain. Power amplifiers maintain this relationship by pushing or pulling current $I$, into or out of the stacks.

From equation 3.3, a stack behaves similar to a capacitor (i.e. $I=c \frac{d V}{d t}$ ). Therefore, as the frequency of $V$ is increased, power amplifiers must supply more current to maintain the relationship $V=S_{P}{ }_{p}$. At high frequencies, power amplifiers will not be able to supply this amount of current and will saturate. To avoid current saturation, low pass prefilters can be placed in series before the power amplifier. In this work, 2-pole Butterworth prefilters with $8 \mathrm{kHz}$ break frequencies were used.

A model of prefilter/power amplifier dynamics is given by

$$
\begin{aligned}
& \frac{d \mathbf{X}_{P}}{d t}=\mathbf{A}_{P} \mathbf{X}_{P}+\mathbf{B}_{P} \mathbf{v}_{P} \\
& \mathbf{V}=S_{p} \mathbf{C}_{p} \mathbf{X}_{P} \\
& \frac{d}{d t} \mathbf{V}=S_{p} \mathbf{C}_{P d} \mathbf{X}_{P} \\
& \text { where } \mathbf{v}_{P}=\left[\begin{array}{llll}
v_{p_{1}} & v_{p_{2}} & \cdots & v_{p_{4}}
\end{array}\right]^{T}, \mathrm{~V}=\left[\begin{array}{llll}
v_{1} & v_{2} & \cdots & \dot{v}_{4}
\end{array}\right]^{T}
\end{aligned}
$$

and $v_{P_{i}}$ is the voltage into the $i^{t h}$ prefilter, $V_{i}$ is the voltage out of the $i^{t h}$ power amplifier, and $S_{p}=100(V / V)$.

\subsection{Anti-aliasing filters and sensors}

Strain at the root of the tool is measured by a set of gages and a bridge network. Strain signals are Frequency Modulated (FM) and broadcasted off of the rotating tool holder by using electro-magnetic transmissions. These transmissions are captured by a receiver, are demodulated, and are fed into anti-aliasing filters. Transmitted and filtered strain signals are then digitized and translated from the rotating coordinate system of the tool holder to the fixed coordinate system of the cartridge and platform.

Since discretization and coordinate translation are commutative, modeling can be simplified by interchanging these two processes. The dynamics of the telemetry system cascaded with anti-aliasing filters, and translated into fixed coordinates can be approximated by 


$$
\begin{aligned}
\overline{d x}_{f t} & =\mathbf{A}_{f} \mathbf{X}_{f}+\mathbf{B}_{f} \varepsilon \\
\mathbf{v}_{\varepsilon} & =S_{f} \mathbf{C}_{f} \mathbf{X}_{f}
\end{aligned}
$$

where $\mathbf{V}_{\varepsilon}$ is a vector of strain voltage signals and $S_{f}$ is the sensitivity of the strain measurement system. Discretization of $\mathbf{V}_{\varepsilon}$ will be shown in section 3.5 .

In this paper, $S_{f}=1.0 \cdot 10^{4}(\mathrm{~V} /(\mathrm{in} / \mathrm{in}))$ and anti-alias filters are five pole Bessel filters with a $8 \mathrm{kHz}$ break frequency.

\subsection{Modified structural dynamics}

From equations 3.3 to 3.6 , a continuous time, modified machine structural dynamic model takes the form,

$$
\begin{aligned}
& \frac{d \mathbf{X}}{d t}=\mathbf{A X}+\mathbf{B f}_{t}+\mathbf{B}_{v} \mathbf{v} p
\end{aligned}
$$

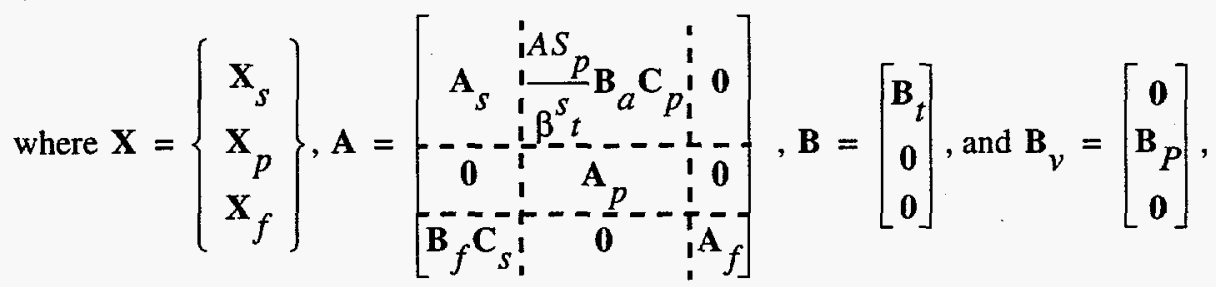

$$
\begin{aligned}
& \mathbf{v}_{\varepsilon}=\mathbf{C}_{V_{\varepsilon}} \mathbf{x} \text { where } \mathbf{C}_{V_{\varepsilon}}=\left[\begin{array}{llll}
\mathbf{0} & \mathbf{0} & S_{f} \mathbf{C}_{f}
\end{array}\right] \text {, } \\
& \mathbf{v}_{p}=\mathbf{C}_{V_{p}} \mathbf{X} \text { where } \mathbf{C}_{V_{p}}=\left[0 S_{p} \mathbf{C}_{p} \mathbf{0}\right] \text {, } \\
& i=\mathbf{C}_{I} \mathbf{X} \text { where } \mathbf{C}_{I}=\frac{A}{\beta^{s}{ }_{t}}\left[h \mathbf{C}_{a d} N_{L} S_{p} \mathbf{C}_{p d} \mathbf{0}\right] \text {, } \\
& \mathbf{d}_{t}=\mathbf{C}_{d_{t}} \mathbf{X} \text { where } \mathbf{C}_{d_{t}}=\left[\begin{array}{lll}
\mathbf{C}_{t} & \mathbf{0} & \mathbf{0}
\end{array}\right],
\end{aligned}
$$

and where $\mathbf{0}$ is a zero matrix place holder of appropriate size.

In discrete time, equation 3.7 is given by

$$
\begin{aligned}
\mathbf{X}(i+1)= & \mathbf{A X}(i)+\mathbf{B}_{t}(i)+\mathbf{B}_{v} \mathbf{v}_{p}(i) \\
\mathbf{v}_{\boldsymbol{\varepsilon}}(i) & =\mathbf{C}_{V_{\boldsymbol{\varepsilon}}} \mathbf{X}(i) \\
\mathbf{v}_{p}(i) & =\mathbf{C}_{V_{p}} \mathbf{X}(i) \\
\boldsymbol{i}(i) & =\mathbf{C}_{I} \mathbf{X}(i) \\
\mathbf{d}_{t}(i) & =\mathbf{C}_{d_{t}} \mathbf{X}(i)
\end{aligned}
$$

where $\mathbf{A}=e^{A T}, \mathbf{B}=\int_{0}^{T} e^{A \tau} d \tau \mathbf{B}, \mathbf{B}_{v}=\int_{0}^{T} e^{A \tau} d \tau \mathbf{B}_{v}{ }^{21}, \mathbf{X}(i)$ is $\mathbf{X}$ at time $t=(i-1) T, \mathbf{f}_{t}(i)$ is $\mathbf{f}_{t}$ at time $t=(i-1) T$, etc....

\subsection{Control law development}

The control law is a mathematical relationship which functionalizes $\mathbf{v}_{p}(i)$ in terms of $\mathbf{V}_{\varepsilon}(i)$. This functionalization couples structural dynamics for the purpose of minimizing modal vibratory response at the tool tip, thereby enhancing depth of cut and maximizing metal removal rate. In this paper, the form of the control law is assumed to be

$$
\mathbf{X}_{c}^{(i+1)}=\mathbf{A}_{C} \mathbf{X}(i)+\mathbf{B}_{C} \mathbf{v}_{\varepsilon}^{(i)}
$$




$$
\mathbf{v}_{p}(i)=\mathbf{C}_{c} \mathbf{X}_{c}(i)
$$

Control law design consists of determining a realization, $\left(\mathbf{A}_{C}, \mathbf{B}_{C}, \mathbf{C}_{c}\right)$, which will robustly minimize $\mathbf{d}_{t}(i)$ while stabilizing the closed loop system.

For the purpose of explication, assume that equation 3.8 is a truth model which represents system dynamics exactly. Also, assume that as a result of system identification there exists a $z$-domain ${ }^{22}$ nominal estimate, $\tilde{G}(z)$, of the dynamics; $\mathbf{G}(z)=\mathbf{C}_{V_{\varepsilon}}(z \mathbf{I}-\mathbf{A})^{-1} \mathbf{B}_{v}$, such that

$$
\begin{gathered}
\left|\tilde{G}_{m n}(z)\right|-\left|\mu_{m n}(z)\right| \leq\left|G_{m n}(z)\right| \leq\left|\tilde{G}_{m n}(z)\right|+\left|\mu_{m n}(z)\right| \\
\angle \tilde{G}_{m n}(z)-\angle \mu_{m n}(z) \leq \angle G_{m n}(z) \leq \angle \tilde{G}_{m n}(z)+\angle \mu_{m n}(z)
\end{gathered}
$$

where $\tilde{G}_{m n}(z)$ and $G_{m n}(z)$ are the $m^{t h}$ row, $n^{t h}$ column elements of $\tilde{G}(z)$ and $\mathbf{G}(z)$ respectively, and $\left|\mu_{m n}(z)\right|$ and $\angle \mu_{m n}(z)$ are $m^{t h}$ row, $n^{t h}$ column magnitude and phase identification uncertainty bounds.

Since $\mathbf{V}_{\varepsilon}(i)$ is related to $\mathbf{d}_{t}(i)$ through bending, minimizing $\mathbf{V}_{\varepsilon}(i)$, will also minimize $\mathbf{d}_{t}(i)$. Therefore, the control law design problem can be restated as to

determine: $\mathbf{H}(z)=\mathbf{C}_{C}\left(z \mathbf{I}-\mathbf{A}_{c}\right)^{-1} \mathbf{B}_{C}$, such that: $(\mathbf{I}+\mathbf{H}(z) \mathbf{P}(z))^{-1}$ is stable and minimal in magnitude for any $\mathbf{P}(z)$ where

$$
\left|\tilde{G}_{m n}(z)\right|-\left|\mu_{m n}(z)\right| \leq\left|P_{m n}(z)\right| \leq\left|\tilde{G}_{m n}(z)\right|+\left|\mu_{m n}(z)\right| \text { and } \angle \tilde{G}_{m n}(z)-\angle \mu_{m n}(z) \leq \angle P{ }_{m n}(z) \leq \angle \tilde{G}_{m n}(z)+\angle \mu_{m n}(z) \text {. }
$$

Symmetry can be used to reduce design complexity. In the frequency range of interest, ovaling of the spindle and bearings is negligible. Therefore, pairs of opposing actuators produce similar forces driven 180 degrees out of phase. This information can be incorporated into the control law up-front for the purpose of limiting design complexity.

If the 1st and 3rd and the 2nd and 4th actuators are opposing pairs, then let $\mathbf{C}_{c}=\mathbf{R} \mathbf{C}_{c_{s y m}}$ where $\mathbf{R}=\left[\begin{array}{cccc}1 & 0 & -1 & 0 \\ 0 & 1 & 0 & -1\end{array}\right]^{T}$. This reduces design complexity from the control of a system with four inputs and two outputs to a system,

$\mathbf{G}(z) \mathbf{R}=\left[\begin{array}{ll}G_{11}(z) & G_{12}(z) \\ G_{21}(z) & G_{22}(z)\end{array}\right]$, with two inputs and two outputs.

Control design complexity can be further reduced by examining the elements of $\mathbf{G}(z) \mathbf{R}$. For the scrutinized system, $\left|G_{11}(z)\right|>8\left|G_{12}(z)\right|,\left|G_{11}(z)\right|>8\left|G_{21}(z)\right|,\left|G_{22}(z)\right|>8\left|G_{12}(z)\right|$, and $\left|G_{22}(z)\right|>8\left|G_{22}(z)\right|$. Therefore, $G(z) \mathbf{R}$ is diagonal dominate. If $\mathbf{C}_{C_{s y m}}\left(z \mathbf{I}-\mathbf{A}_{c}\right)^{-1} \mathbf{B}_{C}=\left[\begin{array}{ll}H_{11}(z) & H_{12}(z) \\ H_{21}(z) & H_{22}(z)\end{array}\right]$, then, due to diagonal dominance, a suitable control law can be designed by letting $H_{12}(z)=H_{21}(z)=0$ and treating the open loop system as two, uncoupled, Single-Input, SingleOutput (SISO) systems with loop gains $\tilde{G}_{11}(z) H_{11}(z)$ and $\tilde{G}_{22}(z) H_{22}(z)$ respectively. Furthermore, for this system, $G_{11}(z) \cong G_{22}(z)$. Therefore, by letting $H_{11}(z)=H_{22}(z)$, the control law design problem can be reformulated from a difficult MIMO solution with uncertainty, to a simplified single loop SISO problem with slightly greater uncertainty. 
Even after employing the above simplifications, control law design is still cumbersome. An initial estimate as to control law dynamics can be determined by using a Linear Quadratic Gaussian (LQG) ${ }^{21}$ control scheme. These dynamics can then be modified using the Nichols chart in the Matlab QFT toolbox ${ }^{24,25}$.

\subsection{Integrated closed loop dynamics}

From equations 3.8 and 3.9, modified machine, tool tip compliance can be computed. Since the objective of control was to make the tool tip dynamically stiff, and since compliance is the inverse of stiffness, the tool tip compliance of the Figure 7 modified machine with control should contain modes with lower amplitudes than the modes in the Figure 4 unmodified machine, tool tip compliance.

Figure 9 shows the magnitude of the Figure 4 unmodified machine, tool tip compliance, the Figure 7 modified machine, tool tip compliance with no control*, and the Figure 7 modified machine, tool tip compliance with control. Unmodified machine compliance contains two dominant modes, whereas modified machine compliance with no control contains only one. This single, modified machine, dominant mode has an amplitude larger than the amplitude of any of the modes in the unmodified machine. Therefore, the modified machine with no control is more flexible and more susceptible to machining instabilities than the unmodified machine. Modified machine dynamics with either PZT and PMN stacks produced similar results.

Also shown in Figure 9 is the tool tip compliance of the modified machine with control. This compliance contains modes which are all low in amplitude. Therefore, the modified machine with control is more rigid and less susceptible to machining instabilities than the modified machine with no control and the unmodified machine.

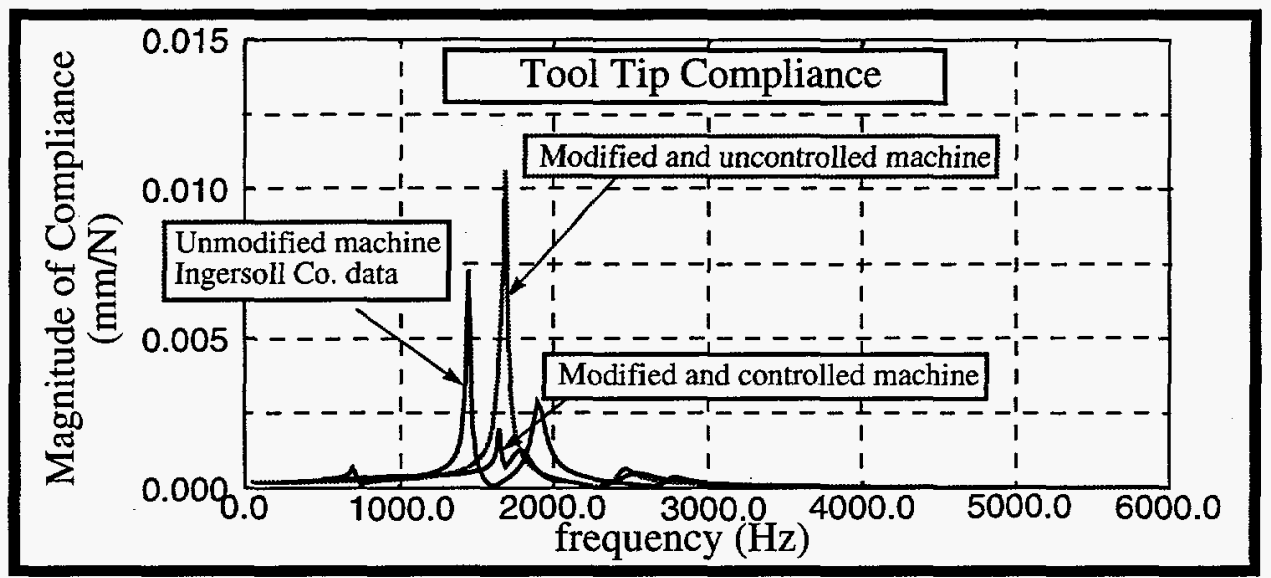

Figure 9: Comparison of tool tip compliances

\subsection{SIMULATION}

Performance enhancement can be described by comparing the stability limits of the unmodified machine, to the stability limits of the modified machine with control. Stability limits were determined by running a series of simulations, and analyzing response data.

Figure 10 is a plot of stability limits for modified and unmodified machines with and without process damping. The specific tool modeled in this simulation extends $80 \mathrm{~mm}$ out from the tool holder, is $16.8 \mathrm{~mm}$ in diameter and has two teeth. In all cases examined, with or without process damping, active control always increased the maximum stable depth of cut. Notice in the cases where there is no process damping, the stable depth of cut is more than doubled. Since the depth of cut is proportional to metal removal rate, the conclusion can be drawn that a successful implementation of this active control strategy can result in more than doubling the metal removal rate of a milling machine.

In sections 2 and 3 , a model of the modified OHM machine was discussed. From a series of simulations, time response and stability data were produced. If time response data cannot be reproduced in hardware, the modified design will not be realizable. Therefore, subcomponent requirements must be evaluated and matched with available hardware. Subcomponent requirements are a bound on hardware operating conditions. Actuators are driven by power limited amplifiers. Therefore, power amplifier

* No control means that $\mathbf{v}_{p}(i)$ is a vector of zeros. 


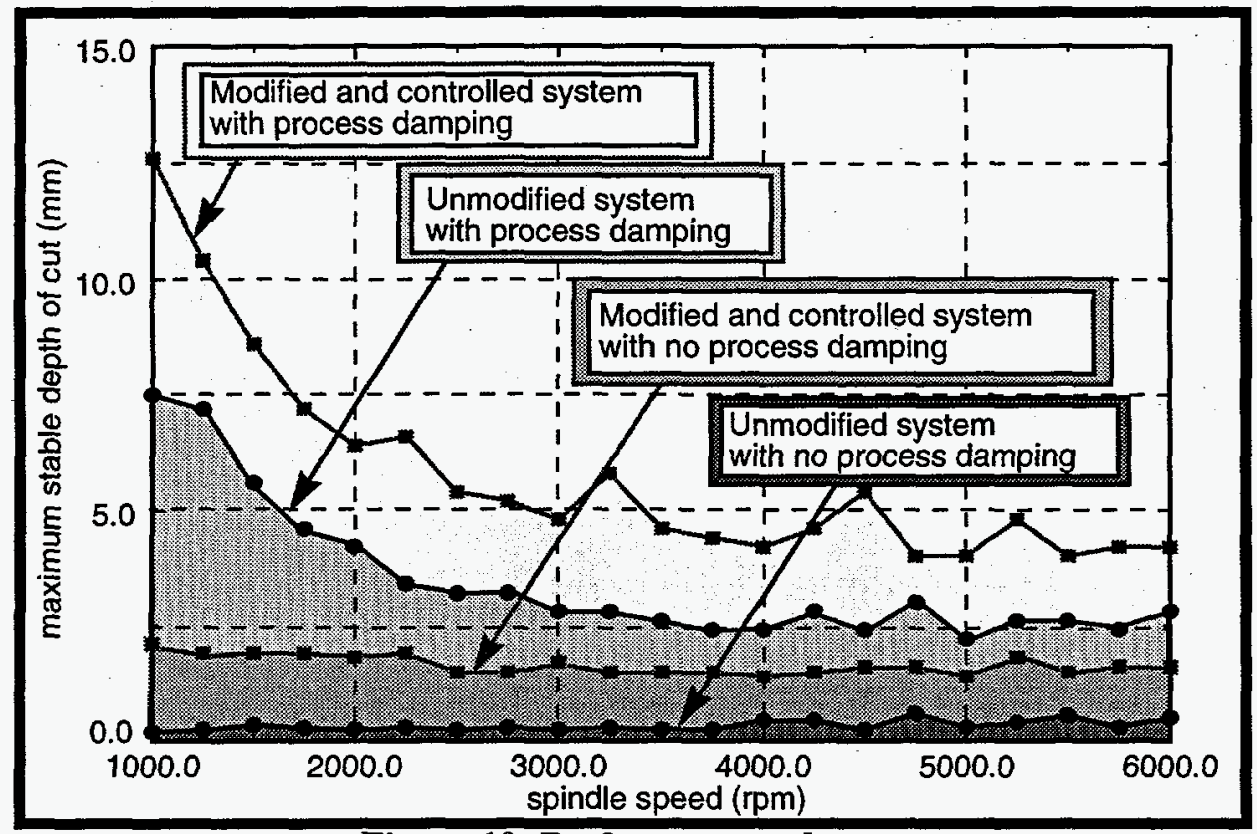

Figure 10: Performance enhancements

current, and voltage requirements must be specified. A/D and D/A converters cannot accept or produce voltages which are above or below limiting values. Therefore, $A / D$ and $D / A$ voltage swing requirements must be specified. Noise exists in any discrete control system, and sensors cannot measure responses below background threshold levels. Therefore, sensor resolution, and quantization bit size requirements must be specified.

Once subcomponent requirements are specified, hardware can be matched to the system. In this study, the subcomponent hardware requirements were determined to match existing hardware or be within the current manufacturing state of the art. Therefore, this active control system is realizable.

\subsection{SUMMATION}

A structural dynamic model of an existing Octahedral Hexapod Milling machine tool was discussed in section 2. Modifications to incorporate an active control system in this model were discussed in section 3 . Finally, in section 4 , results from this simulation are discussed.

Subject to the approximations described in this paper, the computational simulation developed has demonstrated feasibility that an actively controlled spindle system can more than double a conventional milling machines metal removal rate without chatter occurring. This actively controlled or smart spindle is currently being constructed.

\section{ACKNOWLEDGEMENTS}

This work was funded by the Smart Materials and Structures Program, managed by the Defense Advanced Research Project Agency (DARPA), Arlington, VA and contracted through the U. S. Air Force Manufacturing Technology Office, Wright Laboratories, WPAFB, OH. Part of this work was performed at Sandia National Laboratories, which is operated for the U.S. Department of Energy under Contract No. DE-AC04-94AL85000. Also, the authors wish to thank Ingersoll Milling Machine Company for the test data provided.

\section{REFERENCES}

1. Merrit, H.E., "Theory of Self-Excited Machine-Tool Chatter, Contribution to Machine-Tool chatter, Research--1", Journal of Engineering for Industry, Transactions for the ASME, pp. 447-453, November 1965

2. Tobias, S.A., Machine-Tool Vibration, Blackie \& Son Limited, 1965

3. Tlusty, J., Ismail, F., "Special Aspects of Chatter in Milling", Journal of Vibration, Acoustics, Stress, and Reliability in Design, Transactions of ASME, vol. 105, pp. 24-32, January 1983 
4. Shi, H.M., Tobias, S.A., "Theory of Finite Amplitude Machine Tool Instability", International Journal of Machine Tool Design Res., vol. 24, No.1.pp. 45-69, 1984

5. Sadek, M.M., Tobias, S.A., "Reduction of Machine Tool Vibration", American Society of Mechanical Engineering, Applied Mechanics Division, v1, for Meeting, Cincinnati, Ohio, pp. 128-172, September 1973

6. Liu,K.J., Rouch,K.E., “Optimal Cutting Process Stability in Milling Using Active Dynamic Absorber”, DSC-Vol. 38, Active Control of Noise and Vibration, ASME pp. 275-283,1992

7. Tewani,S.G., Switzer,T.C., Walcott,B.L., Rouch, K.E., Massa, T.R., “Active Control of Machine tool Chatter for a Boring Bar: Experimental Results”, DE-vol. 61, Vibration and Control of Mechanical Systems, ASME, pp. 103-115, 1993

8. Lui,K.J., Rouch,K.E., "An Application of Active Dynamic Absorber to the Milling Process and its Experimental Verification", DE-vol. 61 Vibration and Control of Mechanical Systems, ASME, pp. 95-102, 1993

9. Thompson, R.A. "The Modulation of Chatter Vibrations", Journal of Engineering for Industry, Transactions of ASME, pp. 673-677, August 1969

10. Smith, S. Delio, T., "Sensor-Based Chatter Detection and Avoidance By spindle Speed Selection", Journal of Dynamic Systems, Measurement, and Control, vol. 114, pp. 486-492, September 1992

11. Comstock, T.T., Tse, F.S., Lemon, J.R., “Application of Controlled Mechanical Impedance for Reducing Machine Tool Vibrations", Journal of Engineering for Industry, Transactions of the ASME, pp. 1057-1062, November 1969

12. Hong-Yeon Hwang, Jun-Ho Oh, Kwang-Joon Kim, "Modeling and Adaptive Pole Assignment Control in Turning", International Journal of Machine Tools in Manufacturing, vol. 29, No. 2. pp. 275-285, 1989

13. Shiraishi, M., Yamanaka, K., Fujita, H., "Optimal Control of Chatter in Turning", International Journal of Machine Tools in Manufacturing, vol. 31. No.1. pp31-43. 1991

14. Smith, S., Tlusty,J., "An Overview of Modeling and Simulation of the Milling Process", Journal of Engineering for Industry, vol 113,pp. 169-175

15. Redmond, J.M. “Modeling/Simulation for Vibration Control of High Speed Milling Machines" Internal Sandia Memorandum

16. Lauffer,J.P., Hinnerichs, T.D. et. al.,. "Milling Machine for the 21st Century - Goals, Approach, Characterization and Modeling", Smart Structures and Materials, Industrial and Commercial Applications of Smart Structures Technologies, SPIE, vol. 2721, pp. 326-340, 1996

17. Craig, R.R., Structural dynamics, An Introduction to Computer Methods, John Wiley \& Sons, New York, 1981

18. Skelton, R.E., Dynamic Systems Control, Linear Systems Analysis and Synthesis, John Wiley \& Sons, New York, 1988

19. Wilson, O.B. Introduction to Theory and Design of Sonar Transducers, Peninsula Publishing, Los Altos, CA,USA, 1985

20. Hom, C.1., Pilgrim, S.M. et. al., "Calculation of Quasi-Static Electromechanical Coupling Coefficients for Electrostrictive Ceramic Materials", IEEE Transactions on Ultrasonics, Ferroelectrics, and Frequency Control, vol., 41, no.4, July 1994

21. Kwakernaak, H., Sivan, R., Linear Optimal Control Systems, Wiley-Interscience, New York, NY, 1972

22. Oppenheim, A.V., Schafer, R.W., Digital Signal Processing, Prentice-Hall, Inc. Englewood Cliffs, NJ, 1975

23. Ogata,K. Modern Control Engineering, Prentice-Hall,Inc., Englewood Cliffs, NJ., 1970

24. Horowitz, I., Quantitative Feedback Design Theory (QFT), QFT Publications, Boulder, CO, 1993

25. Borghesani, C. Chait, Y., Yaniv, O., Quantitative Feedback Theory Toolbox for use with MATLAB, the MathWorks, Inc., 1994

\section{DISCLAIMER}

This report was prepared as an account of work sponsored by an agency of the United States Government. Neither the United States Government nor any agency thereof, nor any of their empleyees, makes any warranty, express or implied, or assumes any legal liability or responsibility for the accuracy, completeness, or usefulness of any information, apparatus, product, or process disclosed, or represents that its use would not infringe privately owned rights. Reference herein to any specific commercial product, process, or service by trade name, trademark, manufacturer, or otherwise does not necessarily constitute or imply its endorsement, recommendation, or favoring by the United States Government or any agency thereof. The views and opinions of authors expressed herein do not necessarily state or reflect those of the United States Government or any agency thereof. 